\title{
UPPER GASTROINTESTINAL TRACT FOREIGN BODY IN CHILDREN
}

\author{
Shimali Sinha1, Sushant Kumar², Anshumita ${ }^{3}$ \\ ${ }^{1}$ Assistant Professor, Department of Paediatrics, Bhupendra Narayan Mandal University. \\ 2 Professor, Department of Surgery, MGM Medical College, Kishanganj, Bihar. \\ 3Ph. D, Lalit Narayan Mithila University.
}

\section{ABSTRACT}

\section{OBJECTIVE}

Evaluation of foreign body in upper GI tract in children.

\section{STUDY DESIGN}

Retrospective case study.

\section{PLACE AND DURATION}

Mata Gujri Medical College, Kishanganj, Bihar, India, from January 2005 to January 2016.

\section{METHODOLOGY}

Between Jan 2005 to Jan 2016, the record of all children of age group 5-16 were analysed at Mata Gujri Medical College, Kishanganj, Bihar, with a final diagnosis of foreign body in upper GI tract. Data were analysed with respect to demographic data, presenting symptoms, investigations, management and outcome. Age below 5 yrs. and 16 years were excluded from the study.

\section{RESULT}

Total seventy patients were identified (38 boys and 32 girls). Age ranges from 5 yrs. to 16 yrs. Fifty three patients (75.7\%) presented within $24 \mathrm{hrs}$. Thirteen patients (18.6\%) had underlying predisposing factors. The most common FB found in 30 patients $(42.8 \%)$ was coin. The most common symptoms were drooling of saliva in 42 patients (60\%) followed by vomiting (51.4\%) in 36 patients. Followup ranges from one week to six months and all were recovered without any sequelae.

\section{KEYWORDS}

Upper Gastrointestinal FB, Flexible Upper GI Scope, Children.

HOW TO CITE THIS ARTICLE: Sinha S, Kumar S, Anshumita. Upper gastrointestinal tract foreign body in children. J. Evolution Med. Dent. Sci. 2016;5(71):5161-5164, DOI: 10.14260/jemds/2016/1170

\section{INTRODUCTION}

Foreign body ingestion is a common problem all over the world with $80 \%$ of the case reported in children.(1) Most swallowed foreign bodies pass harmlessly through the gastrointestinal (GI) tract. Foreign bodies that damage the GI tract, become lodged or have associated toxicity must be identified and removed. Children with pre-existing GI abnormalities (e.g., tracheoesophageal fistula, stenosing lesions, previous GI surgery) are at an increased risk for complications. Foreign bodies in the air and food passages are the sixth most common cause of accidental death in the United States. Diagnosis of foreign body ingestion is difficult specially when there is no witness of ingestion of $\mathrm{FB}(2,3)$. Various type of foreign body was documented depending upon the country, culture, medical records, etc. In paediatric population, toddlers younger than 5 years are most commonly affected because of their increased mobility and natural propensity for experimentation. Although, children younger than 6 months are rarely able to get a foreign object into the oropharynx. Infants can ingest foreign bodies with

Financial or Other, Competing Interest: None.

Submission 12-06-2016, Peer Review 19-07-2016,

Acceptance 25-07-2016, Published 03-09-2016.

Corresponding Author:

Dr. Sushant Kumar,

Professor, Department of Surgery,

MGM Medical College,

Kishanganj, Bihar.

E-mail: sushantkumar2002@yahoo.com

DOI: $10.14260 /$ jemds $/ 2016 / 1170$ the assistance of a sibling. Although, any child can swallow a foreign body and most incidents result in minor annoyance; however, some can become a challenging problem and have serious life-threatening complications. Flexible Upper GI scope is recommended in all cases as gold standard of the treatment. It is safe, effective and well-demonstrated. However, it is costlier, requires the presence of a skilled endoscopist, necessitates sedation or general anaesthesia may require the subsequent observation or hospitalisation. Generally, Rat tooth forceps, snare, balloon, etc. are used for removal of FB. This study was conducted to review our experience in terms of type of foreign body, its demographic, and its management and followup.

\section{METHODOLOGY}

Retrospective analysis of 70 patients came to Mata Gujri Medical College, Kishanganj, Bihar, between January 2005 to January 2016 between 5-16 yrs. of age. All case below 5 yrs. and above 16 yrs. were excluded from the study. Flexible Upper gastrointestinal endoscopy is also not very convenient in the patients below $5 \mathrm{yrs}$. of age. Most of the patients were presented in casualty within 24 hrs. of ingestion of FB while others presented themselves at surgery, ENT and Paediatric OPD. Data were collected from record room and analysed in terms of demography, symptoms, investigations, management and outcomes. Details of history, physical examinations and investigations was analysed with the documents available at record room. Proper consent form was checked for both diagnostic as well as therapeutic removal of the foreign body. 
Particular emphasis given over sign and symptoms and presenting complaint. Most of the time during diagnostic upper gastrointestinal endoscopy, consultant is able to do curative management of the patients by removing the FB in same sitting of the procedure. Whatever be the material removed is identified and preserved. A proper documentation of the object removed is made and finally handed over to the relatives. Proper safety is maintained while removing sharp edged object to prevent any damage during its path of removal. If any damage occurred, then it was assessed and managed conservatively. Due to this, patient can be retained for some time in the hospital and looked for any further complications. Flexible upper GI scope (Olympus) was used to diagnose and treat all cases under local or general anaesthesia. Rat tooth forceps, snare, magnet, balloon, etc. are used to remove all types of FB.

\section{RESULT}

Total seventy patients were admitted with a diagnosis of upper GI foreign body. Age ranges from 5 yrs. to 16 yrs. There were 38 boys and 32 girls. All patients were presented within one hour to six months of ingestion of FB. Fifty three patients were $(75.7 \%)$ presented within 24 hrs. of ingestion. Most common symptoms were drooling of saliva in 30 patients (60\%) followed by vomiting in 36 patients (51\%). Six patients were asymptomatic. Thirteen patients (18.6\%) had underlying predisposing factor like poor socioeconomic condition, lack of education, poor hygiene, etc. Foreign body removed were bolus in 10 patients, metallic object in two patients and coin in one patient. All were presented within 24 hrs. with drooling of saliva and dysphagia.

Detailed history, physical examinations, chest, abdominal and neck radiograph, CT scan and flexible upper GI endoscopy were performed for proper diagnosis and plan of management. Sign and symptoms varies according to the position, type and duration of the object. Foreign body were located in oesophagus in 33 pat patients, in hypopharynx in 7 patients and in stomach in 20 patients. Others had FB distal to second part of duodenum, which can be assessed with negative upper GI scopy and can with the help of radiological investigations or proper history of passage of FB from faeces.

Various type of FB was removed. The most common FB was coin (42.8\%). Other type was metallic objects (ear ring, safety pin, and hair clip), food bolus, fish bone, disc batteries, plastic materials and glasses. Length of hospital stay was 1 to 12 days. All patients were completely recovered without any complications or sequelae.

Most of the patients need no treatment after removal of FB. Others need prophylactic antibiotic of 3-5 days with analgesics particularly in cases with sharp pointed type of FB. Sometime minor tear noted in tract while pulling out FB and needs conservative management.

\begin{tabular}{|c|c|c|}
\hline Type of FB & Number & Percentage \\
\hline Coin & 30 & $42.8 \%$ \\
\hline Metal & 14 & $20 \%$ \\
\hline Food Bolus & 10 & $14.3 \%$ \\
\hline Fish Bone & 6 & $8.6 \%$ \\
\hline Disc Battery & 3 & $4.3 \%$ \\
\hline Plastic Materials & 2 & $2.9 \%$ \\
\hline Marble Piece & 2 & $2.9 \%$ \\
\hline Glass Piece & 1 & $1.4 \%$ \\
\hline Cotton & 1 & $1.4 \%$ \\
\hline No FB Found & 1 & $1.4 \%$ \\
\hline \multicolumn{2}{|c|}{ Type of Foreign Body Ingestion } \\
\hline
\end{tabular}

Patients presented with wide range of symptoms and sign depending upon age, nature of $\mathrm{FB}$, anatomical site of lodgement and length of time since ingestion. ${ }^{1}$ Vomiting, dysphagia, drooling of saliva, and respiratory symptoms were the most common presenting symptoms.2,3,4 In our study, drooling of saliva was the most common presenting complaints. Type of FB ingested differs among countries according to feeding habits, culture, festivals, socioeconomic status, etc.5,6,7 Several studies show that the coin is the most common type of FB found to be ingested. $(8,8)$ In our study, coin is the most common type of FB found in upper GI tract followed by fish bone and metal piece. $(3,4,5,6,7,8,9,10)$ Food bolus was found to be more common in previous stricture of

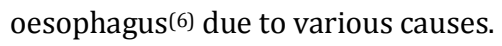

\begin{tabular}{|c|c|c|}
\hline Symptoms & $\begin{array}{c}\text { Number of } \\
\text { Patients }\end{array}$ & $\mathbf{\%}$ \\
\hline Drooling of Saliva & 42 & 60 \\
\hline Vomiting & 36 & 51 \\
\hline Respiratory Symptoms & 31 & 44 \\
\hline Dysphagia & 23 & 33 \\
\hline Odynophagia & 6 & 9 \\
\hline Asymptomatic & 6 & 9 \\
\hline Weight Loss & 1 & 1.4 \\
\hline \multicolumn{2}{|c|}{ Presenting Complaint of FB Ingestions } \\
\hline
\end{tabular}

Lin et al(9) conclude that diagnosis of FB ingestion based on three important elements: eye witness, $\mathrm{x}$-ray and upper GI endoscopy. Several study showed that use of radiograph $(10,8,10,11)$ is useful tool in the diagnosis of $\mathrm{FB}$ ingestions but radiolucent substance can be missed by this method. Luk et al(12) showed that CT scan is $96 \%$ specific in diagnosis of FB even in negative upper GI endoscopy cases. In general, x-ray is baseline investigation for FB ingestions.

Most children who have swallowed a foreign body do not require specialised care. For the large majority, providing comfort care while transporting to an emergency department is all that is required. Patients with drooling may require suction and proper attention. Children benefit by being allowed to remain with their parents and being allowed to assume a position of comfort. Although, a theoretical risk of spontaneously vomiting and then aspiration of a foreign body exists, this is unusual. Children should not routinely be intubated to protect their airways. Similarly, do not attempt to dislodge a foreign body from a spontaneously breathing patient by giving abdominal thrusts or syrup of ipecac. The usual goal of upper gastrointestinal FB management is to localise the position of the ingested foreign body. Patients with drooling, marked emesis or altered mental status (likely from excess vagal stimulation) may require supportive measures to protect the airway.

Most patients should undergo radiographic imaging like X-ray, USG, CT scan, etc. Metal detectors maybe used to locate metallic foreign bodies. Even radio-opaque foreign bodies maybe difficult to localise. Referral for endoscopy should be considered.

Remember that children with no symptoms may have impacted foreign bodies and that children with foreign body sensation or pain may not. Radiographs of about $15 \%$ of children presenting to the hospital after witnessed coin ingestions do not show a coin. Although, some will have vomited or otherwise removed the ingested object before 
their evaluation. This suggests that not all children with even witnessed foreign body ingestions have truly ingested something.

So, many methods were used to remove FB from upper GI tract through flexible upper GI scope such as rat tooth forceps, balloon extractor, snare, magnet, etc. The choice of instrument depends upon surgeon's choice(8) and available instruments. We prefer rat tooth forceps and snare most of the time. FB which can damage upper GI tract should be identified and removed quickly as early as possible.(12) Waltzman et al(12) reported that around $25-30 \%$ FB ingested will pass spontaneously without complications. Lin et al(11) and Kamath et al(13) noted that FB should be removed as soon as possible to avoid complications as compared to our study.

Complication related to FB ingestion is uncommon, but maybe life-threatening sometime.(7) Longstanding FB have higher incidence of complications like perforation, obstruction, peritonitis, abscess, fistula formations, etc. Type of FB ingested is related to the outcome of the patients. (14) Most common FB cause complications are food (29\%), coin (29\%), and batteries.(14) Timely diagnosis and management is necessary to avoid complications.

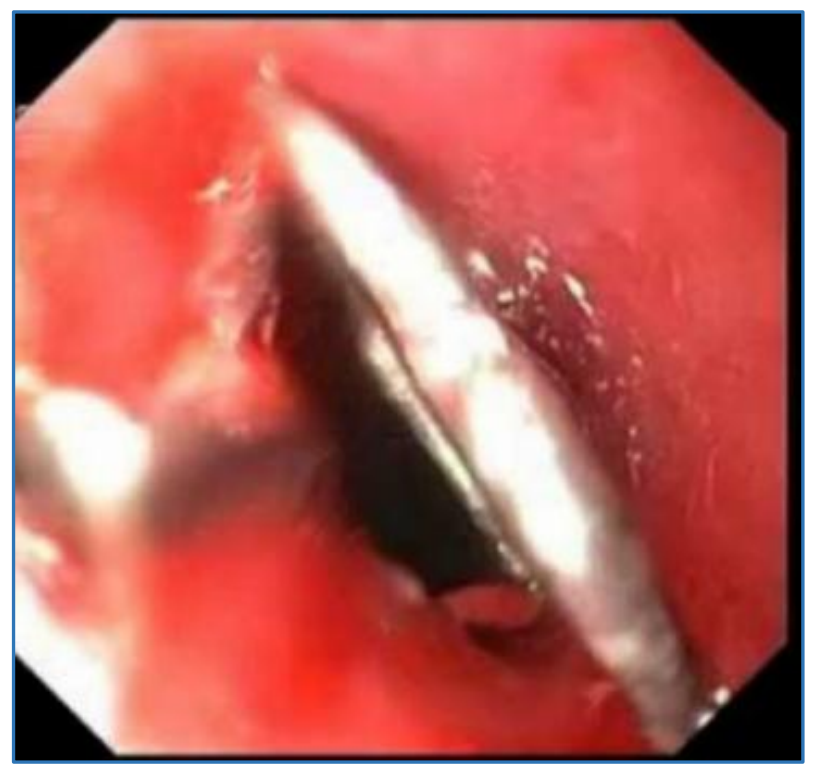

Fig. 1: Coin inside Oesophagus

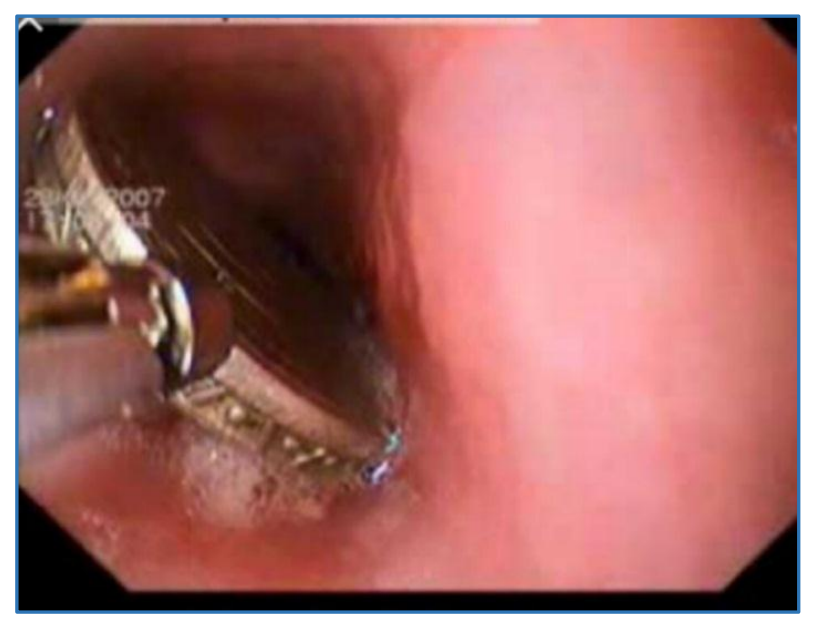

Fig. 2: Removal of Coin

\section{DISCUSSION}

Paediatric foreign body ingestion is a worldwide problem. Impaction of swallowed fish bones is more commonly observed in countries where fish is a major dietary staple including Asian countries. A massive database describing paediatric foreign body injury in European and other countries, the "Susy Safe project," recently published information regarding nearly 17,000 cases in children aged 14 years and younger; about $18 \%$ of these involved foreign body ingestion. All children regards of age can ingest FB, but more common in younger patients.(15) Most of the FB can travel its course harmlessly.(4) Most of the patients were below 10 yrs. (51\%) as compared with other data.(1,3,4) A witness history of FB ingestion is extremely important for quick diagnosis.(5) Louie et al(5) published a study of 225 case studies with majority of their patients having witnessed history of FB ingestions. In our study, a witnessed history of FB ingestion was found in $74.2 \%$ of the cases. Upper oesophagus is narrow part, so most of the FB was found in this part.(3) In our study, majority of the FB were found in upper oesophagus $(83 \%)$ as compared with other study. $(3,6)$ Coin is the most common type of FB found followed by metal piece, food bolus, fish bone, etc.

\section{CONCLUSION}

Foreign body ingestion is common problem in children and usually an accidental event. Long retained FB is associated with higher incidence of complications. Flexible upper GI scope is best tool to deal with this problem as it will diagnose as well as treat the problem.

\section{REFERENCES}

1. Saki N, Nikakhlagh S, Safai F, et al. Oesophageal foreign bodies in children. Pak J Med Sci 2007;23:854-6.

2. Louie JP, Alpern ER, Windreich RM. Witnessed and unwitnessed esophageal foreign bodies in children. Pediatr Emerg Care 2005;21(9):582-5.

3. Zhang S, Cui Y, Gong X, et al. Endoscopic management of foreign bodies in the upper gastrointestinal tract in South China: a retrospective study of 561 cases. Dig Dis Sci 2010;55(5):1305-12.

4. Lai AT, Chow TL, Lee DT, et al. Risk factors predicting the development of complications after foreign body ingestion. Br J Surg 2003;90(12):1531-5.

5. Orji FT, Akpeh JO, Okolugbo NE. Management of esophageal foreign bodies: experience in a developing country. World J Surg 2012;36(5):1083-8.

6. Higo R, Matsumoto Y, Ichimura K, et al. Foreign bodies in the aerodigestive tract in pediatric patients. Auris Nasus Larynx 2003;30(4):397-401.

7. Pak WM, Lee CW, Fung KH, et al. A prospective study of foreign body-ingestion in 311 children. Int J Pediatr Otorhinolaryngol 2001;58(1):37-45.

8. Little DC, Shah SR, St Peter SD, et al. Esophageal foreign bodies in the pediatric population: our first 500 cases. J Pediatric Surg 2006;41(5):914-8.

9. Lin $\mathrm{CH}$, Chen AC, Tsai JD, et al. Endoscopic removal of foreign bodies in children. Kaohsiung J Med Sci 2007;23(9):447-52.

10. Shinhar SY, Strabbing RJ, Madgy DN. Esophagoscopy for removal of foreign bodies in the pediatric population. Int J Pediatr Otorhinolaryngol 2003;67(9):977-9.

11. Luk WH, Fan WC, Chan RY, et al. Foreign body ingestion: comparison of diagnostic accuracy of computed 
tomography versus endoscopy. J Laryngol Otol 2009;123(5):535-40.

12. Waltzman ML, Baskin M, Wypij D, et al. A randomized clinical trial of the management of esophageal coins in children. Pediatrics 2005;116(3):614-9.

13. Kamath $\mathrm{P}$, Bhojwani KM, Prasannaraj $\mathrm{T}$, et al. Foreign bodies in the aerodigestive tract-a clinical study of cases in the coastal belt of South India. Am J Otolaryngol 2006;27(6):373-7.
14. Gregori D, Scarinzi C, Morra B, et al. Ingested foreign bodies causing complications and requiring hospitalization in European children: result from ESFBI study. Pediatr Int 2010;52(1):26-32.

15. Wyllie R. Foreign bodies in the gastrointestinal tract. Curr Opin Paediatr 2006;18(5):563-4. 